\title{
Bright Young Star Candidates in the Rosette Nebula
}

\author{
P. S. Chiang, W. P. Chen, J. Z. Li \\ Graduate Institute of Astronomy, National Central University, \\ Chung-Li 32054, Taiwan \\ Y.-H. Chu \\ Department of Astronomy, University of Illinois, Urbana-Champaign, \\ $I L, 61801, U S A$
}

\begin{abstract}
We identified possible optical young stars in the Rosette Nebula by using the IRAS and the ROSAT databases. The previously reported "diffuse" X-ray emission has been resolved into point sources. Forty-seven point sources are detected above 3-sigma of the X-ray background, and twenty-seven of these have stellar counterparts. Some of the young star candidates appear to be associated Herbig-Haro nebular features.
\end{abstract}

\section{The Rosette Nebula}

The Rosette Nebula (NGC 2237) is a prominent H II region currently expanding due to the radiation pressure and stellar winds from the $\mathrm{OB}$ association in NGC 2244 located near the center. The Nebula has an angular extent of $\sim 1.5$ degrees, which at a distance of $1.5 \mathrm{kpc}$ (Pérez 1991), corresponds to a physical size of more than about $40 \mathrm{pc}$.

\section{The Young Star Candidates}

The Rosette Nebular has been observed with the ROSAT PSPC and HRI instruments. IRAS sources in the region have been reported by Cox et al (1990). To identify possible pre-main sequence stars, we compiled a list of sources detected by both ROSAT and IRAS in the vicinity of the Rosette Nebula. The combination of X-ray and infrared observations to search for young stars has been successful in nearby molecular clouds, such as in Taurus, Ophiuchus, Chamaeleon, and Lupus (see the review by Feigelson \& Montmerle 1999). Because Rosette Nebula is some 10 times further away than these molecular clouds, only the brightest IRAS and ROSAT objects in Rosette Nebula could have been detected. Our analysis has resolved previous reported "diffuse" X-ray emission into point sources. A total of 47 point sources are detected at 3 -sigma above the background. Here we report only sources detected in both catalogs. Optical counterparts have been sought within 1' of the nominal IRAS and ROSAT positions in the H-alpha image taken by $\mathrm{T}$. Rector et al. (private comm.) and the Digitized Sky Survey images. Infrared and X-ray sources without optical counterparts are possibly embedded sources (Feigelson \& Montmerle 1999).

Table 1 lists 5 IRAS/ROSAT sources thus identified. These should be studied to confirm their pre-main sequence nature. Fig. 1 shows an example of such an IRAS/ROSAT source, for which the $\mathrm{H}_{\alpha}$ image is compared with the 

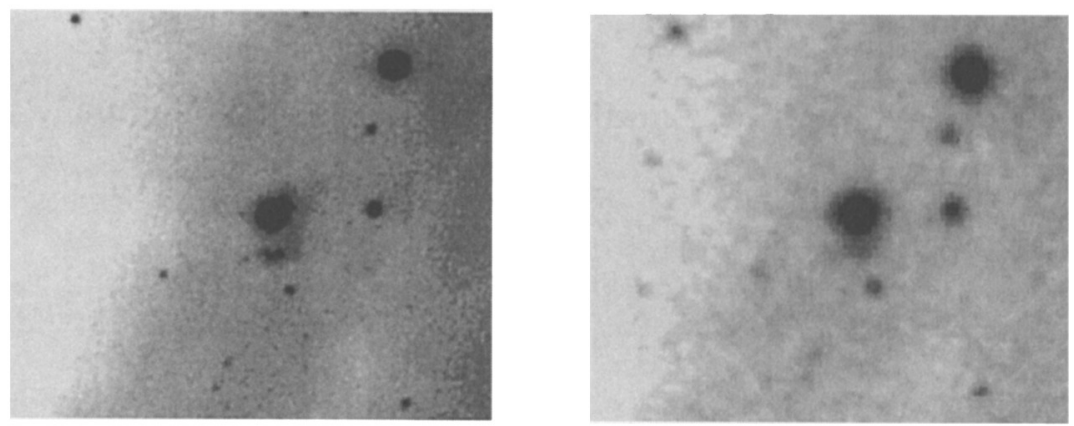

Figure 1. The H-alpha (left) and Digitized Sky Survey (right) images of Object 1 in Table 1. Each image is about 90 " on a side, with north to the top and east to the left.

Digitized Sky Survey image. An emission nebulosity, possibly of Herbig-Haro object nature, is readily seen to the south of, but may or may not be associated with, the bright optical star (No. 1 in Table 1). A full papaer of our results will be published soon.

Table 1: Bright Young Star Candidates in the Rosette Nebula

\begin{tabular}{cccccc}
\hline No. & IRAS & $\begin{array}{c}\text { ROSAT/RA DEC } \\
(2000)\end{array}$ & $\begin{array}{c}\text { USNO/RA DEC } \\
(2000)\end{array}$ & $\mathrm{m}_{\mathrm{B}} \mathrm{m}_{\mathrm{R}}$ \\
\hline 1 & $06288+0456$ & $063129.7+045450$ & $063129.8+045449$ & 12.416 .4 \\
& & & $063128.4+045450$ & 19.219 \\
\hline 2 & $06288+0452$ & $063133.2+045052$ & $063131.5+045100$ & 10.516 .2 \\
& & $063133.3+045047$ & $063134.9+045100$ & 2019.7 \\
& & $063133.7+045035$ & $063136.1+045100$ & 16.318 .3 \\
& & & $063130.0+045059$ & 18.418 .9 \\
& & & $063133.4+045038$ & 9.412 .3 \\
\hline 3 & $06289+0504$ & $063138.2+050131$ & $063138.4+050137$ & 8.411 .1 \\
& & $063138.4+050142$ & $063140.5+050136$ & 20.419 .9 \\
& & & $063140.7+050136$ & 20.220 .1 \\
& & & $063141.7+050136$ & 20.519 .2 \\
\hline 4 & $06290+0508$ & $063139.7+050558$ & $063141.8+050606$ & 19.519 .4 \\
& & $063139.9+050548$ & $063143.5+050549$ & 12.815 .4 \\
& & $063140.3+050546$ & $063138.9+050540$ & 20.919 .7 \\
& & & $063140.0+050557$ & 12.515 .1 \\
& & & $063141.8+050550$ & 18.919 .1 \\
& & & $063142.7+050545$ & 20.119 .6 \\
& & & $063143.5+050549$ & 12.815 .4 \\
\hline 5 & $06298+0444$ & $063231.0+044237$ & $063231.4+044234$ & 16.417 .5 \\
\hline
\end{tabular}

\section{References}

Feigelson, E. D. \& Montmerle, T. 1999, ARA\&A, 37, 363

Cox, P., Deharveng, L., \& Leene, A. 1990, A\&A, 230,181

Pérez, M. R., 1991, RMxAA, 22, 99 